\title{
Utilization of a Swine (Sus scrofa) Model for Lipid Emulsion Resuscitation Studies
}

\author{
Carrie Crane, Eileen Sagini, Arthur D. Johnson, and Joseph O’Sullivan \\ AMEDD Center and School, Academy of Health Sciences Graduate School, 3490 Forage Road, Dunlap Hall, \\ Fort Sam Houston, TX 78234, USA \\ Correspondence should be addressed to Joseph O’Sullivan, joseph.osullivan@amedd.army.mil
}

Received 24 September 2012; Accepted 5 November 2012

Academic Editors: J. P. Estebe, D. Karakaya, A. Mizutani, D. E. Raines, and A. Wiebalck

Copyright (๑) 2012 Carrie Crane et al. This is an open access article distributed under the Creative Commons Attribution License, which permits unrestricted use, distribution, and reproduction in any medium, provided the original work is properly cited.

Background. There have been some discussions that using swine or rabbits in a resuscitation model to study lipid emulsion infusions may be inappropriate because of a consistent "pseudoallergic" reaction that has been found in lipid-based nanoparticle liposome drug carrier systems. Assertions have been made that the lipid emulsions may contain a certain amount of liposomes; therefore swine may not be an appropriate model for study. Methods. This study was prospective, crossover design within subjects, and research design with each Yorkshire swine receiving a 20\% lipid bolus infusion followed by a lipid infusion for $10 \mathrm{~min}$. Each swine had a total of four blood draws and each draw had complete blood gas analysis with three different types of inflammatory markers examined. Cardio vascular monitoring was performed every 2 minutes. Results. Using data reported in similar studies, a large effect size of 0.6 was calculated. Using the effect size of 0.6 , a power of 0.8 and an alpha of 0.05 it was determined that a sample size of five swine was needed. There were no significant changes in any CV parameter both before and after lipid emulsion. Likewise there were no significant changes in any of the blood tests, nor any inflammatory markers. Conclusions. There were no significant changes in the examined parameters with swine before and after lipid emulsion infusions. It is suggested that due to the close size of swine to humans, similar physiology, and ease of using these animals, they may be utilized for lipid emulsion studies.

\section{Introduction}

There have been many antidotal letters indicating that an accidental overdose of local anesthetics (or other lipid drugs) has been treated effectively with a $20 \%$ lipid emulsion infusion [1-6]. These patients were not responding effectively to standard ACLS resuscitation protocols but recovered shortly after a last ditch effort with a lipid emulsion infusion [7]. However, a comparison of different resuscitation techniques with and without lipid emulsion infusion has never been performed to determine if lipid emulsion or ACLS protocol or possibly a combination of the two is the most effective treatment [8]. The model that may be the easiest to determine effectiveness of lipid emulsion in the face of drug overdose is swine. Swine (specifically Sus scrofa) physiology, size, and ease of use seem to make these animals very compatible models for this type of research [9]. Rats are not the best model due to more anatomical and physiological variance with humans and the inherent variability in using humans for CPR compressions (fatigue) given no current mechanical device for these small animals.

Controversy exists using the swine model for lipid emulsion resuscitation testing. Issues arose when nanoparticulate vehicle systems were being investigated to increase the therapeutic index of many drugs (Doxil, Taxol, and Ambisome). Often the vehicle of choice for these drugs is liposomes or micelles. Imbedding these drugs inside these specialized fat molecules greatly increases their duration of action and effectiveness. Unfortunately, these liposomes (or micellar solvents) have been documented to cause a "Cactivation-related pseudoallergy" (CARPA) [10, 11]. This CARPA effect consistently leads to cardiovascular collapse and laboratory abnormalities. Swine have been documented as being the best model to observe this negative CARPA affect [12]. Minute amounts of multilamellar vesicles or liposomes can cause anaphylactoid shock with "major hemodynamic changes" in swine, which is easily reproducible [13]. Documented consistent hemodynamic changes include 
declines in SBP (hypotension) and CO with ECG changes such as tachycardia, bradycardia, ST segment and T wave changes, and ventricular fibrillation. This evidence gives rise to criticism that swine (Sus scrofa) should not be used with lipid emulsion studies because the swine may have a pseudoallergic (CARPA) reaction to $20 \%$ lipid emulsion infusion (liposomes?) [14]. This consistent large effect size from previous liposomes or micellar solvent studies allow us to rule out this CARPA effect with a minimum number of swine. This effect seems to be consistent with all species of swine $[11,12]$.

Our 3 research questions are as follows. Is there a statistical difference before and after lipid emulsion infusion relative to three inflammation markers (IgE, IgG, and Creactive protein)? Is there a statistical difference before and after lipid emulsion infusion relative to cardiovascular parameters $\left(\mathrm{EtCO}_{2}, \mathrm{TV}, \mathrm{RR}, \mathrm{ECG}, \mathrm{SpO}_{2}, \mathrm{VHR}, \mathrm{SBP}, \mathrm{DBP}\right.$, MAP, CO, and core temperature)? Lastly, is there a statistical difference before and after lipid emulsion infusion relative to blood gas analysis $\left(\mathrm{pH}, \mathrm{PaCO}_{2}, \mathrm{PaO}_{2}, \mathrm{Na}^{+}, \mathrm{K}^{+}, \mathrm{Cl}^{-}, \mathrm{iCa}^{+}\right.$, $\mathrm{Hct}, \mathrm{Hgb}, \mathrm{O}_{2}$ Sat, and $\left.\mathrm{BE}\right)$ ?

\section{Materials and Methods}

2.1. Study Design. This study was a prospective, crossover within subjects design. The Institutional Animal Care and Use Committee (IACUC) approved the protocol. The animals received care in compliance with the Animal Welfare Act and the Guide for the Use of Laboratory Animals.

2.2. Setting. Five male Yorkshire-Cross (Sus scrofa) swine weighing $55-80 \mathrm{~kg}$ were acclimated for at least 3 days to ensure good state of health, fed a standard diet, and remained NPO after midnight the day of the experiment. The weight and size of the swine approximates the average weight of an adult male. In addition, male swine were used to decrease possible confounding variables related to female hormones.

2.3. Procedure. Anesthesia was induced with an intramuscular injection of Telazol $(4-8 \mathrm{mg} / \mathrm{kg})$, Atropine $(0.05 \mathrm{mg} / \mathrm{kg})$, Isoflurane $(2-4 \%)$, and oxygen (100\%). After intubation, Isoflurane was reduced to $1-2 \%$ for the remainder of the experiment. A peripheral IV catheter was started with a continuous infusion of Lactated Ringer's solution at $0.5 \mathrm{cc} / \mathrm{kg} / \mathrm{min}$. The animals were ventilated (tidal volume $8-$ $10 \mathrm{cc} / \mathrm{kg}$, respiratory rate $8-14$ breaths per minute) with a standard Narkomed anesthesia machine (Draeger, Telford, PA, USA) with the ventilation parameters the same during the procedure. All swine were continuously monitored with the following standard monitors: HR, SBP, MAP, DBP, ECG, $\mathrm{SpO}_{2}, \mathrm{CO}, \mathrm{EtCO}_{2}$, Isoflurane end tidal concentration, and rectal temperature. The left carotid artery was cannulated with a 20G angiocatheter using a cut-down technique. Likewise a large bore venous catheter was inserted into the left jugular vein. These two central catheters were used for blood sampling and fluid/lipid emulsion infusions and attached to the Marquette Solar 800 system for continuous $\mathrm{BP}$ pressure monitoring. Another arterial catheter was inserted into the femoral artery and attached to the Vigileo cardiac output monitor (Edwards Lifesciences) for cardiac output monitoring.

2.4. Methods. The experiment was conducted in 2 phases. In phase one, the swine were instrumented and allowed to stabilize before lipid infusion. Baseline VS documented with blood gas analysis, and inflammatory markers (C-reactive, protein, IgG, and $\operatorname{IgE}$ ) were drawn. In the second phase, $20 \%$ lipid emulsion (Fresenius Kabi, Germany), IV was given over 2-3 minutes $(2 \mathrm{cc} / \mathrm{kg})$, and lipid emulsion infusion at $0.25 \mathrm{cc} / \mathrm{kg} / \mathrm{min}$ is started and infused for $20 \mathrm{~min}$. At the conclusion of the lipid infusion, the timer is started to mark the 15-, 30- and 45-minute intervals at which time blood draws are initiated. VS and CO were documented every 2 minutes.

2.5. Materials. Blood pressure was monitored by the use of a carotid arterial line connected to the Marquette Solar 800 Medical System. It is precise within $1 \mathrm{~mm} \mathrm{Hg}$ and accurate within $2 \mathrm{~mm} \mathrm{Hg}$. A RadioShack stopwatch was used to measure the time for stabilization, blood draws and lipid infusions, and the increments in blood pressure. The stopwatch is precise within one hundredth of a second and accurate within one tenth of a second.

\section{Results}

The minimum number of animals was used to obtain a statistically valid result. Using the effect size of 0.6 , a power of 0.8 , and an alpha of 0.05 , it was determined that a sample size of five swine was needed for this study. A MANOVA was used to analyze the pretest variables of all laboratory values, weight, vital signs, and NPO deficit replacement. There were no statistically differences between the groups $(P>0.05)$, indicating that all 5 swine were equivalent within these parameters. All swine were similar in weight and size (mean $58.8 \mathrm{~kg}$, SD 8.6).

It is worthy of note that 4 of the 5 swine exhibited a red, blotchy, and cutaneous transitory mottling, primarily on the abdomen, almost immediately after the lipid infusion. There was no change in cardiovascular parameters, temperature, or changes in blood tests after the appearance of the rash. The mottling almost totally disappeared after 10-15 minutes with no ill effects noted.

The current dose of lipid emulsion for local anesthetic toxicity as determined by the Association of Anesthetists of Great Britain and Ireland (AAGBI) [15] and Weinberg [16] as the standard dose that researchers and clinicians have determined to be effective for resuscitation of a toxic level of local anesthetics is $1.5 \mathrm{cc} / \mathrm{kg}$ of $20 \%$ lipid emulsion administered over $1 \mathrm{~min}$, followed by an infusion of $15 \mathrm{cc} / \mathrm{kg} / \mathrm{hr}$. (or $0.25 \mathrm{cc} / \mathrm{kg} / \mathrm{min}$ ) for $20-30 \mathrm{~min}$. AAGBI suggests not infusing more than $12 \mathrm{cc} / \mathrm{kg}$ total dose and Weinberg suggests a maximum of $8 \mathrm{cc} / \mathrm{kg}$. For this study, we will be using a bolus of $20 \%$ lipid emulsion of $2 \mathrm{cc} / \mathrm{kg}$ as it is within suggested standards of $1-3 \mathrm{cc} / \mathrm{kg}$ that many successful published studies have utilized $[17,18]$. 
TABLE 1: Blood gas analysis: means and standard deviations.

\begin{tabular}{lcccc}
\hline Parameter & Baseline & 15 minutes & 30 minutes & 45 minutes \\
\hline $\mathrm{O}_{2}$ Sat & $100 \pm 0$ & $100 \pm 0$ & $99.80 \pm 0.45$ & $100 \pm 0$ \\
$\mathrm{pH}$ & $7.45 \pm 0.027$ & $7.45 \pm 0.03$ & $7.42 \pm 0.027$ & $7.46 \pm 0.03$ \\
Sodium & $140 \pm 2.6$ & $139 \pm 3.2$ & $137 \pm 3.7$ & $138 \pm 2.5$ \\
Chloride & $104 \pm 3.46$ & $100 \pm 2.3$ & $101 \pm 2.0$ & $100 \pm 1.15$ \\
$\mathrm{iCa}$ & $1.31 \pm 0.15$ & $1.32 \pm 0.07$ & $1.30 \pm 0.079$ & $1.30 \pm 0.075$ \\
$\mathrm{Hct}$ & $31.6 \pm 3.2$ & $33.2 \pm 1.38$ & $31.6 \pm 1.49$ & $30.9 \pm 2.27$ \\
$\mathrm{HgB}$ & $10.14 \pm 1.1$ & $10.9 \pm 0.64$ & $10.44 \pm 0.38$ & $10.1 \pm 0.49$ \\
$\mathrm{PaO}_{2}$ & $518.4 \pm 72.2$ & $523 \pm 65.67$ & $500.4 \pm 16.22$ & $523 \pm 17.9$ \\
$\mathrm{BE}$ & $4.18 \pm 2.4$ & $4.1 \pm 0.90$ & $5.96 \pm 3.1$ & $6.4 \pm 3.0$ \\
\hline
\end{tabular}

TABLE 2: Cardiovascular parameters: means and standard deviations.

\begin{tabular}{lcccc}
\hline Parameter & Baseline & 15 minutes & 30 minutes & 45 minutes \\
\hline EtCO $_{2}$ & $38 \pm 3.2$ & $42 \pm 3.3$ & $41 \pm 3.9$ & $41 \pm 3.24$ \\
$\mathrm{RETRNTV}_{\mathrm{SpO}_{2}}$ & $455 \pm 138.65$ & $464 \pm 133.7$ & $464 \pm 132.91$ & $465 \pm 132$ \\
$\mathrm{VHR}$ & $99.40 \pm 0.54$ & $99.00 \pm 0.70$ & $99.2 \pm 0.83$ & $99.0 \pm 1.22$ \\
$\mathrm{SBP}$ & $100 \pm 12.03$ & $107 \pm 13.71$ & $126 \pm 25.5$ & $114 \pm 6.4$ \\
$\mathrm{DBP}$ & $105 \pm 11.2$ & $130 \pm 36.28$ & $101 \pm 23.82$ & $100 \pm 12.7$ \\
$\mathrm{MAP}$ & $74 \pm 9.2$ & $93 \pm 24.5$ & $69 \pm 18.12$ & $72 \pm 22.7$ \\
$\mathrm{CO}$ & $82.6 \pm 11.6$ & $108.4 \pm 27.8$ & $81.2 \pm 19.01$ & $82 \pm 17.4$ \\
\hline
\end{tabular}

3.1. Inflammatory Markers. A repeated ANOVA was used to determine if there were significant differences between the baseline (no lipids), 15, 30, and 45 minutes after administration of lipids. There were no significant differences between the baseline, 15-minute, 30-minute, and 45-minute IgG levels $(P>0.05)$. The means and standard deviations and analyses of IgG were as follows: baseline ranged from 397 to 802 (mean $=571 \pm 168.120) ; 15$ minute ranged from $378-$ $801($ mean $=577 \pm 181.5) ; 30$ minute ranged from 398 to 822 $($ mean $=579.8 \pm 180.31) ; 45$ minute ranged from 389 to 813 (mean $=572.2 \pm 182)$. There were no significant differences between baseline, 15-minute, 30-minute, and 45-minute IgE and C-reactive protein levels $(P>0.05)$. All levels for IgE were $<0.5$, and all levels from $C$-reactive protein were $<0.3$.

3.2. Blood Gas Analysis. A repeated ANOVA was used to determine if there were significant differences of the blood gas analysis between the baseline (no lipids), 15, 30, and 45 minutes after administration of lipids (see Table 1). There were no significant differences over time in the following: $\mathrm{O}_{2}$ saturation $(P=0.374)$; $\mathrm{pH}(P=0.190)$; sodium $(P=$ $0.428)$; potassium $(P=0.376)$; chloride $(P=0.334)$; calcium $(P=0.918) ;$ Hct $(P=0.448) ; \operatorname{Hgb}(P=0.333) ; \mathrm{PA} 02$ $(P=0.562) ; \mathrm{BE}(P=0.072)$.

3.3. Cardiovascular Parameters. A repeated ANOVA was used to determine if there were significant differences in cardiovascular parameters between the baseline (no lipids), 15, 30, and 45 minutes after administration of lipids (See Table 2). There were no significant differences over time in the following: $\operatorname{EtCO}_{2}(P=0.554)$; retrntv $(P=0.675)$; RR
$(P=0.465) ; \mathrm{SpO}_{2}(P=0.681) ; \operatorname{VHR}(P=0.054) ; \mathrm{SBP}$ $(P=0.334) ; \operatorname{DBP}(P=0.359) ; \operatorname{MAP}(P=0.338) ; \mathrm{CO}$ $(P=0.549)$.

\section{Discussion}

We initiated three different types of blood tests to monitor inflammation markers. These tests may demonstrate a difference if any true allergic reaction is occurring. Type I, type II and generalized inflammation markers used were IgE, IgG, and C-reactive protein, respectively. These tests were chosen as markers for inflammatory response to test for an allergic response as each of them is indicative of a different type of allergic reaction.

$\mathrm{IgE}$ is a type I-mediated immediate multisystem reaction. When allergens bind to the IgE bound to membrane receptors on mast cells, potent vasoactive mediators such as leukotrienes, histamine, and prostaglandins are released. The vasodilation and increased capillary permeability causes anaphylactic shock symptoms including hypertension, hypotension, hypoxemia, urticaria, cyanosis, fever, and metabolic acidosis. This is usually an immediate hypersensitivity reaction.

IgG is a type II antibody-mediated cytotoxic reaction. This type of reaction is described as an antibody reaction with an antigen on a cell or tissue. This antigenantibody reaction results in activation of cytotoxic $\mathrm{T}$ cells or macrophages as well as the $\mathrm{C}$ cascade, with resulting cytolysis or phagocytosis of cells. Examples of this type of reactions include transfusion reactions or drug induced. This Type II blood test is also known as the cytotoxic, or antibody-dependent reaction and is also used for immune 
complex diseases. This test usually takes minutes to hours for measurable effects.

C-reactive protein (CRP) is an acute phase protein. Following infection or any tissue injury from trauma, burns or any inflammatory cause, serum CRP concentrations rise rapidly and significantly. It is not specific. A high result is only a general indication of acute inflammation. CRP levels at more than 1000 times greater than normal have been found. The extent of tissue injury and the course of the ensuing illness can be measured by the increase in CRP levels. Unfortunately, it has been found that CRP is able to bind to phospholipids, and this property may affect the results in this experiment [19].

The other blood tests measured standard blood gas analysis. These included, $\mathrm{pH}, \mathrm{PaCO}_{2}, \mathrm{PaO}_{2}, \mathrm{Na}^{+}, \mathrm{K}^{+}, \mathrm{Cl}^{-}$, $\mathrm{iCa}^{+}$, Hct, Hgb, $\mathrm{O}_{2}$ Sat, and BE. These tests were within subjects to determine any changes in these parameters before and after lipid infusion.

Lastly, and most importantly, cardiovascular and ventilation monitoring consisted of $\mathrm{EtCO}_{2}, \mathrm{TV}, \mathrm{RR}, \mathrm{ECG}, \mathrm{SpO}_{2}$, VHR, SBP, DBP, MAP, CO, and core temperature. These data were collected every 2 minutes to determine any changes within animals before and after lipid infusion. Because of the profound consistent cardiovascular effect of CARPA with liposomes on the swine model, this was the most important testing parameter.

Continued research and advancement in treating toxic overdoses of drugs requires an acceptable model for research design. This study demonstrates that the use of this species of swine may be an acceptable model for lipid emulsion (at $2 \mathrm{cc} / \mathrm{kg}$ with an infusion of $15 \mathrm{cc} / \mathrm{kg} / \mathrm{hr}$ ) research based on laboratory blood gas and cardiovascular parameters. In the duration of our experiment $(45 \mathrm{~min})$, there was no statistically significant change in any of the selected inflammation markers in swine. The cause and impact of the transitory red, mottling, and cutaneous rash are not known. However, there were no significant changes in any cardiovascular parameters during the visualization of the red mottling. The side effects of delivering large doses of lipid emulsions were investigated by Hiller and team [20]. They determined, in rats, that following a high volume of $20 \%$ lipid infusion, the histology was normal, and the mean LD50 was $67 \mathrm{cc} / \mathrm{kg}$ which is one order of magnitude higher than standard doses. Of course, this cannot be transferred to the swine model directly, but does bode well for a safe therapeutic index for lipid emulsion infusions [21].

The central question remains as to why CARPA was not observed in these studies, despite assertions that swine may have a CARPA reaction to lipid emulsions [14]. It is assumed, because of the literature that confirms that swine are very sensitive to liposomes/phospholipid assemblies or liposomes combined with drug formulations [10-12], that lipid emulsion infusions do not trigger CARPA. As little as 5-10 mg of liposomes cause major hemodynamic changes in swine while under general anesthesia [10-12]. None of swine had any hemodynamic effect before or after lipid emulsion infusion. One issue not addressed in this study is that maybe very low or very high doses of lipid emulsions may trigger the CARPA response. Our dose $(2 \mathrm{cc} / \mathrm{kg})$ was the current therapeutic clinical dose for lipid emulsion infusion which appears to be safe in a swine model. The formulation for our lipid emulsion does not appear to contain liposomes of which swine are very sensitive. Currently, it is animal studies and individual case reports which have guided the use of lipid emulsion infusions in treating local and nonlocal anesthetic drug toxicities. Future animal studies will help shape the use of lipid emulsion infusions for possible treatment of other drug toxicities.

\section{Conclusion}

Small animal models, computer models, or isolated organ models are not effective techniques to study toxic overdose with comparative resuscitation techniques and lipid emulsion infusions. In vivo, large animal models are the key to understanding the interactions of drugs, major organs, ACLS protocols, pharmacokinetics, and pharmacodynamics due to the complex interactions of these systems. Resuscitation studies using small animal models have the risk of providing fatigue and variability of CPR/BLS technique. We have shown with this within-subjects design that there is no significant difference before or after lipid emulsion infusions in cardiovascular changes, inflammatory markers, nor blood gas analysis using this breed of swine. Based on this evidence, we believe that the use of this breed of swine is an acceptable model to utilize for comparative resuscitation in lipid emulsion infusion studies. The size of this study dictates that we cannot legitimately exclude other breed-specific problems as there can be a great variation in response to different populations. Therefore, future studies utilizing other breeds of swine or higher doses of lipid emulsions may be necessary as the results may differ.

\section{Funding}

This work received funding from Tri-Service Nursing Research Program, Bethesda, MD, USA.

\section{References}

[1] H. Ludot, J. Y. Tharin, M. Belouadah, J. X. Mazoit, and J. M. Malinovsky, "Successful resuscitation after ropivacaine and lidocaine-induced ventricular arrhythmia following posterior lumbar plexus block in a child," Anesthesia and Analgesia, vol. 106, no. 5, pp. 1572-1574, 2008.

[2] S. D. H. Finn, D. R. Uncles, J. Willers, and N. Sable, "Early treatment of a quetiapine and sertraline overdose with Intralipid®," Anaesthesia, vol. 64, no. 2, pp. 191-194, 2009.

[3] A. J. Sirianni, K. C. Osterhoudt, D. P. Calello et al., "Use of lipid emulsion in the resuscitation of a patient with prolonged cardiovascular collapse after overdose of bupropion and lamotrigine," Annals of Emergency Medicine, vol. 51, no. 4, pp. 412-415.e1, 2008.

[4] G. Foxall, R. Mccahon, J. Lamb, J. G. Hardman, and N. M. Bedforth, "Levobupivacaine-induced seizures and cardiovascular collapse treated with Intralipid," Anaesthesia, vol. 62, no. 5, pp. 516-518, 2007.

[5] M. Harvey, G. Cave, and A. Kazemi, "Intralipid infusion diminishes return of spontaneous circulation after hypoxic 
cardiac arrest in rabbits," Anesthesia and Analgesia, vol. 108, no. 4, pp. 1163-1168, 2009.

[6] S. Markowitz and J. M. Neal, "Immediate lipid emulsion therapy in the successful treatment of bupivacaine systemic toxicity," Regional Anesthesia and Pain Medicine, vol. 34, no. 3, p. 276, 2009.

[7] G. Weinberg, "Lipid rescue resuscitation from local anaesthetic cardiac toxicity," Toxicological Reviews, vol. 25, no. 3, pp. 139-145, 2006.

[8] L. Rothschild, S. Bern, S. Oswald, and G. Weinberg, "Intravenous lipid emulsion in clinical toxicology," Scandinavian Journal of Trauma, Resuscitation, and Emergency Medicine, vol. 18, article 51, 2010.

[9] F. Dorandeu, J. R. Mikler, H. Thiermann et al., "Swine models in the design of more effective medical countermeasures against organophosphorus poisoning," Toxicology, vol. 233, no. 1-3, pp. 128-144, 2007.

[10] J. Szebeni, "Complement activation-related pseudoallergy caused by liposomes, micellar carriers of intravenous drugs, and radiocontrast agents," Critical Reviews in Therapeutic Drug Carrier Systems, vol. 18, no. 6, pp. 567-606, 2001.

[11] J. Szebeni, "Complement activation-related pseudoallergy: a new class of drug-induced acute immune toxicity," Toxicology, vol. 216, no. 2-3, pp. 106-121, 2005.

[12] J. Szebeni, C. R. Alving, L. Rosivall et al., "Animal models of complement-mediated hypersensitivity reactions to liposomes and other lipid-based nanoparticles," Journal of Liposome Research, vol. 17, no. 2, pp. 107-117, 2007.

[13] J. Szebeni, L. Baranyi, S. Sávay et al., "Complement activationrelated cardiac anaphylaxis in pigs: role of $\mathrm{C} 5 \mathrm{a}$ anaphylatoxin and adenosine in liposome-induced abnormalities in ECG and heart function," American Journal of Physiology, vol. 290, no. 3, pp. H1050-H1058, 2006.

[14] B. Zider and G. Weinberg, "Are pigs the right model for lipid resuscitation?” AANA Journal, vol. 79, no. 6, pp. 453-454, e453.

[15] The Association of Anaesthetists of Great Britain and Ireland 2010, Guidelines for the Management of Sever Local Anaesthetic Toxcity, 2012, http://www.aagbi.org/sites/default/ files/la_toxicity_2010_0.pdf.

[16] G. Weinberg, "Lipid rescue: caveats and recommendations for the 'Silver Bullet," Regional Anesthesia and Pain Medicine, vol. 29, pp. 74-75, 2004.

[17] R. J. Litz, M. Popp, S. N. Stehr, and T. Koch, "Successful resuscitation of a patient with ropivacaine-induced asystole after axillary plexus block using lipid infusion," Anaesthesia, vol. 61, no. 8, pp. 800-801, 2006.

[18] M. A. Rosenblatt, M. Abel, G. W. Fischer, C. J. Itzkovich, and J. B. Eisenkraft, "Successful use of a 20\% lipid emulsion to resuscitate a patient after a presumed bupivacaine-related cardiac arrest," Anesthesiology, vol. 105, no. 1, pp. 217-218, 2006.

[19] X. Caide, Z. Liu, Q. Gao, Q. Zhou, and S. F. Sui, "Specific interaction of rabbit C-reactive protein with phospholipid membranes," Thin Solid Films, vol. 284-285, pp. 793-796, 1996.

[20] D. B. Hiller, G. Di Gregorio, K. Kelly et al., "Safety of high volume lipid emulsion infusion: a first approximation of LD50 in rats," Regional Anesthesia and Pain Medicine, vol. 35, no. 2, pp. 140-144, 2010.

[21] M. Buhl, J. Gjedsted, A. Granfeldt et al., "Circulating free fatty acids do not contribute to the acute systemic inflammatory response. An experimental study in porcine endotoxaemia,"
Basic and Clinical Pharmacology and Toxicology, vol. 105, no. 5, pp. 319-326, 2009. 


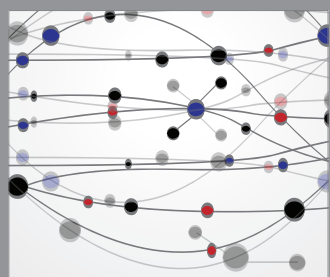

The Scientific World Journal
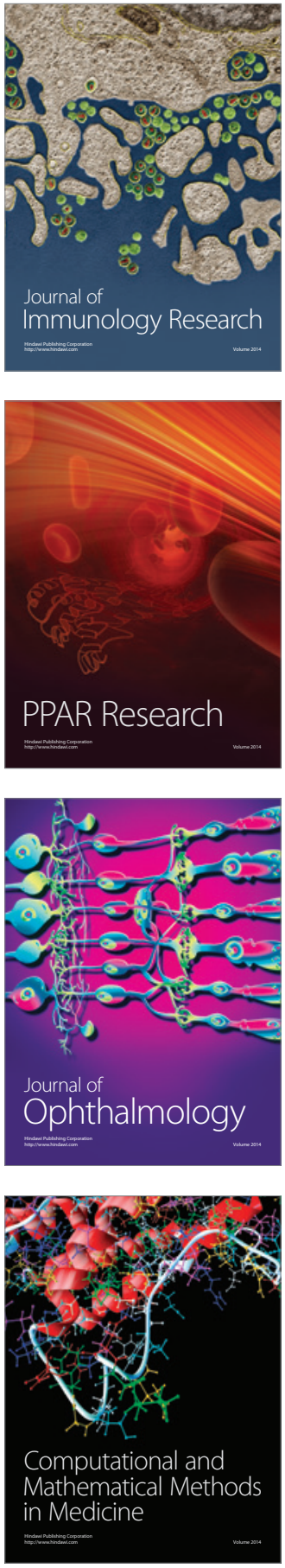

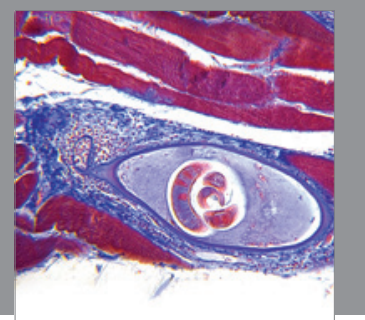

Gastroenterology

Research and Practice
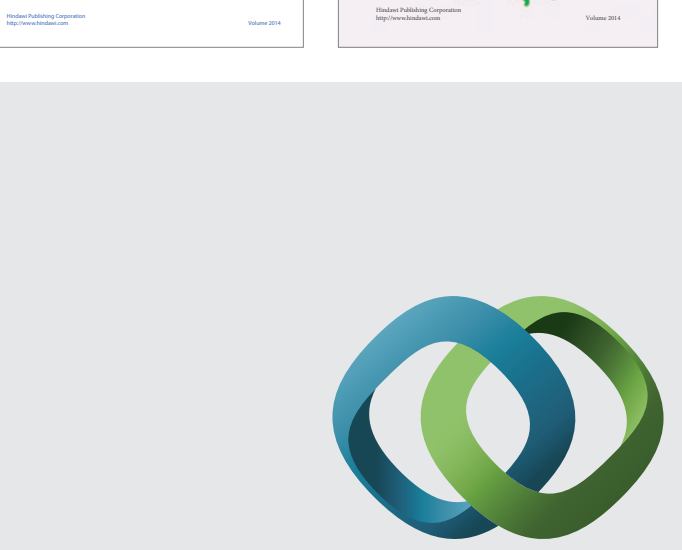

\section{Hindawi}

Submit your manuscripts at

http://www.hindawi.com
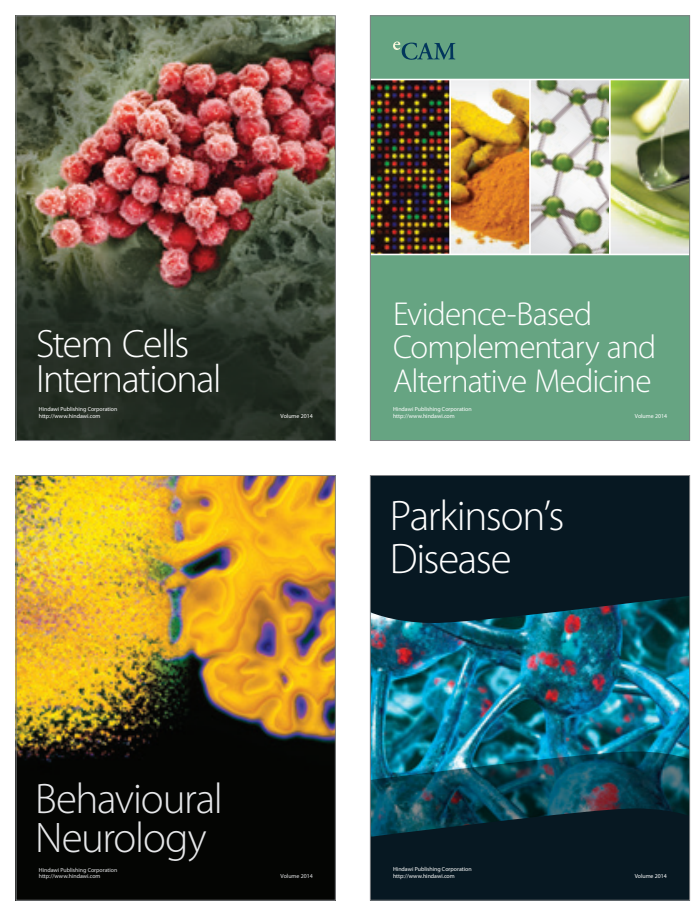

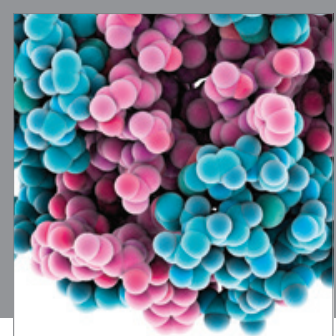

Journal of
Diabetes Research

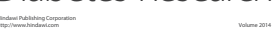

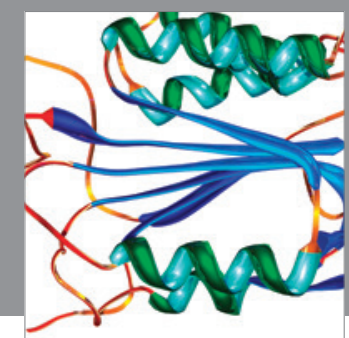

Disease Markers
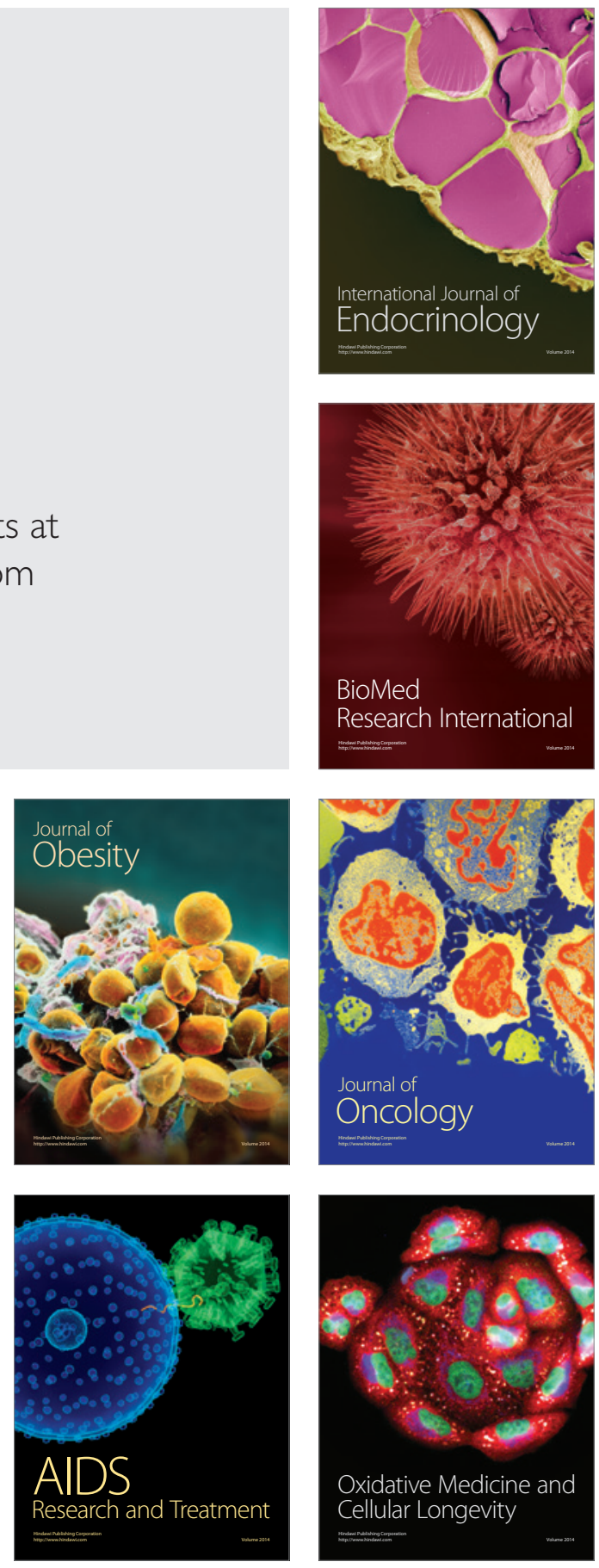